\title{
Autoimmune response in AIDS
}

SIR-The viral protein Nef has an important role in the often long latency period of infection by the human immunodeficiency virus HIV-1. Antibodies raised against Nef have been detected in otherwise seronegative individuals ${ }^{1}$ and $\mathrm{T}$-cell mediated responses to Nef have been reported ${ }^{2}$. Even during the latency period, when only $a$
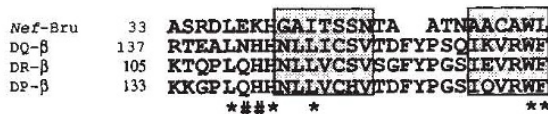
«\#\#\# *

$b$

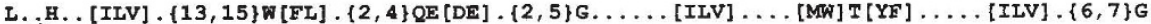
Li...

[ENQ] [HK]

a, Amino-acid sequences of an HIV-1 Nef protein and HLA class II antigen DQ, DR and DP $\beta$-chains are displayed showing the region of similarity. Asterisks, positions containing common or conservative amino acids in all HLA class II $\beta$-chains and HIV-1 Nef sequences (except Mal). The \# sign indicates an additional set conserved in all but a single unusual HLA class II DO $\beta$-chain Boxes indicate the postulated $\beta$ strands modelled on the $\alpha 3$ domain of the HLA class I protein $b$, Common pattern to HLA class II $\beta$-chain and Nef HIV-I pattern expressed as a regular expression. Dot, any amino acid; capital letters in square brakets, conserved alternative amino acids; curly brakets, range of a variable spacer or gap. (Further details available on request from T.F.S.)

a small proportion of peripheral blood lymphocytes $\left(1\right.$ in $\left.10^{4}-10^{5}\right)$ appear to express viral structural messenger RNA, a progressive impairment of the immune defences is observed ${ }^{3.4}$. This last observation is compatible with an autoimmune response as part of the progression of disease. We have found a common structural pattern in the sequences of Nef and the $\beta$-chain of the HLA class II histocompatibility antigens which could induce such an autoimmune response.

The HIV-1 Nef proteins share a region of 60-70 amino acids which is highly conserved. A search of the SWISS-PROT protein sequence database (release 12 , EMBL, 1989) reveals that this region is similar to the immunoglobulin-like second extracellular domain of the $\beta$-chain of the HLA class II histocompatibility antigens (see figure). Within this region, a common diagnostic pattern can be identified such that no other known proteins in this region are compatible with the postulated HLA class $\beta$-chain structure modelled on the known $\alpha 3$ domain of the HLA class I protein.

We identified this common pattern using an iterative multialignment pattern induction algorithm ${ }^{5}$, and find it is statistically equivalent to sharing more than 10 identical amino acids. The sequence contains some of the amino acids thought to be essential for the HLA class II $\beta$-chain fold and most of the common amino acids are distributed proximally on the surface of the HLA class II $\beta$-chain/ $\alpha 3$ domain model structure. (We chose the placement of gaps in the pattern to be compatible with the modelled structure.) The pattern appears in all HLA class II position 61-63, removing an otherwise conserved tryptophan. The pattern is not found in HIV-2 or simian immunodeficiency virus sequences equivalent to Nef.

The existence of this common structural pattern and the early generation of antibodies against Nef suggests a mechanism for an autoimmune response against the HLA class II $\beta$-chain induced by HIV-1 Nef through 'molecular mimicry'. This possibility is further supported by reports ${ }^{6.7}$ of antibodies against self-HLA class II molecules and lymphocytes in individuals infected with HIV-1. It is relevant that animals injected with antibodies directed against their constitutive class II histocompatibility molecules develop a severe immunodeficiency comparable to that present in the HIV-infected human individuals.

Finally, the absence of the HLA/Nef common pattern in HIV-2 Nef is consistent with the greatly reduced pathogenicity observed for this subtype. An immune response against Nef during the latent phase of the infection, resulting in an autoimmune response against HLA class II molecules, could lead to the progressive deterioration of the immune system. This impairment appears to allow eventual active replication, leading to the late stages of AIDS. These hypotheses, if

\footnotetext{
1. Ameisen, J. et al. Aids Res. Hum. Retroviruses 5, 279291 (1989).
}

2. Gluckman, J.C. et al Abstr. Int. AIDS Meeting, Canada 547 (1989)

3. Miedema, F. et al. J. clin. Invest. 82, 1908-1914 (1988)

4. Terpstra, F.G. et al. Eur. J. Immun. 19, 667-673 (1989).

5. Smith, R., \& Smith, T.F. Proc. natn Acad. Sci. U.S.A. 87 118-122 (1990)

6. Golding, H. et al. J. exp. Med. 167, 914-923 (1988).

7. Dorsett, B., Cronin, W., Chuma, V. \& loachim, H.L. Am. J Med. 78, 621-625 (1985) confirmed, suggest early therapeutic intervention aimed at neutralizing the adverse effects of Nef during the latent phase of the disease.

Department of Tumor Virology,

Miguel A. VegA*

RODERIC GUIGÓ

TEMPLE F. SMITH

Molecular Biology Computer Research

Resource,

Department of Biostatistics,

Dana-Farber Cancer Institute Harvard

School of Public Health,

Boston,

Massachusetts 02115, USA

* Present address: Centro de Biologia. Universidad Autónoma de Madrid, Cantoblanco, Spain.

\section{Drought resistance in rice}

SIR-Drought is a major problem for much of the world's rice crop. Of the 146 million hectares of land on which rice is grown, only one half is irrigated. In the rest, which accounts for one-quarter of rice-grain production, yields are often depressed by drought ${ }^{1.2}$. Moreover, in many irrigated rice soils, hard pans block root growth and so impede water extraction ${ }^{3}$.

The impact of drought on crop production can be reduced by developing varieties better adapted to water deficits ${ }^{4}$ Despite this, drought resistance in rice has historically had a low priority in breeding programmes $^{2}$. As in many other crops ${ }^{5}$, two causes of this are lack of basic understanding of the mechanisms of drought injury and resistance, and want of ready means to connect physiological and biochemical knowledge with genetics and breeding. Participants at a recent workshop ${ }^{6}$ considered how plant biotechnology could help to relieve these constraints. The following conclusions were reached.

(1) Especially for tropical rices, there is an urgent need to understand the plant better, in relation to drought at various life-cycle stages, and to physiological, biochemical and molecular levels of organization.

(2) The genetic material and the research expertise available in the international rice research network in Asia are great resources. Building bridges between this network and plant scientists in other countries could help to speed the development of new tools for drought resistance breeding, and to advance basic knowledge of water stress responses in rice.

(3) New breeding tools would be provided primarily by incorporating selected physiological traits into the restrictionfragment length polymorphisms program being developed by the Rockefeller Foundation's International Program on Rice Biotechnology?. An example would be the genes governing deep rooting/ 